Proceedings of the II Symposium on Applied Nuclear Physics and Innovative Technologies, Kraków, September 24-27, 2014

\title{
Dark Photon Searches with the KLOE Detector
}

\author{
C. BLOISE* on behalf of the KLOE-2 Collaboration \\ Laboratori Nazionali di Frascati dll'INFN, Via Fermi 40, 00044 Frascati, Italy
}

\begin{abstract}
The KLOE-2 experiment at the Frascati Laboratory has recently obtained several results on dark photon searches. We present published analyses and the preliminary results of the study of the final states with $\mathrm{e}^{+} \mathrm{e}^{-} \gamma$, and $\mu^{+} \mu^{-}$plus missing energy.
\end{abstract}

DOI: 10.12693/APhysPolA.127.1565

PACS: $13.66 . \mathrm{Hk}$

\section{Introduction}

The gravitational and cosmological evidence of dark matter (DM) as the dominant, $85 \%$ fraction of the matter in the Universe still remains, after 50 years of investigation, the only firm experimental evidence of DM, although some puzzling measurements from astroparticle experiments could be explained in terms of DM scattering on nuclei or DM annihilation. The thermal relic abundance of DM implies small couplings with the SM, main feature of the standard WIMP scenario, whose DM candidate is a weakly interacting massive particle (WIMP) with mass from $10-10^{4} \mathrm{GeV}$. Some non-WIMP DM models postulate the existence of a dark gauge symmetry $\mathrm{U}(1)_{\mathrm{D}}$ [1] giving rise to a secluded sector with mediating bosons (dark photons), fermions, and scalar fields for the spontaneous breaking of the symmetry (dark higgs fields). In general, the phenomenology relating to the dark sector, especially in the case $m_{\mathrm{U}}<m_{\mathrm{DM}}$, is really different from the standard WIMP scenario. Some of the secluded sectors can be tested at the $\mathrm{e}^{+} \mathrm{e}^{-}$colliders searching for vector or scalar particles at the $\mathrm{GeV}$ scale [2]. A DM sector mediated by a light dark photon with mass $m_{\mathrm{U}} \leq 1 \mathrm{GeV}$ could explain both, the positron excess measured by the balloon-borne experiments HEAT [3] and PAMELA [4] and by AMS-02 on the International Space Station [5], and the measurement of the antiproton flux [6] that is instead in agreement with the abundance expected from secondary production in the interstellar medium (ISM) interactions of the primary cosmic rays. In this case, the kinetic mixing parameter $\epsilon$ of the dark photon with the $\gamma / \mathrm{Z}$ boson is expected to be of $O\left(10^{-4}-10^{-2}\right)$, a range of couplings that can be studied at the $\phi$ and B factories. A light dark photon could be observed in a variety of final states, among which are: (i) sharp resonance at $m_{\mathrm{U}}$ in the invariant mass distribution of charged lepton or pion pairs, in $\mathrm{e}^{+} \mathrm{e}^{-} \rightarrow \gamma l^{+} l^{-}$or $V \rightarrow \mathrm{Pl}^{+} l^{-}$reactions, where $V(P)$ stands for any vector (pseudoscalar) meson, and $l^{ \pm}$for muons, electrons or charged pions; (ii) dark-higgsstrahlung processes [7],

${ }^{*}$ corresponding author; e-mail: Caterina.Bloise@lnf.infn.it $\mathrm{e}^{+} \mathrm{e}^{-} \rightarrow \mathrm{Uh}^{\prime}$, giving rise to different final states, relating with the $m_{\mathrm{U}} / m_{\mathrm{h}^{\prime}}$ masses and branching fractions. In particular, for $m_{\mathrm{h}^{\prime}}<m_{\mathrm{U}}$, the light dark higgs signature is a peaking missing mass associated with a peaking invariant mass in the light dark photon decay channel, $\mathrm{U} \rightarrow l^{+} l^{-}$. The cross-section depends on $\alpha_{\mathrm{D}} \times \epsilon^{2}$, where $\alpha_{\mathrm{D}}$ is the coupling constant in the dark sector.

\section{Dark photon searches with KLOE}

The KLOE experiment, at the Frascati $\phi$-factory, has searched for dark photon production using the final states: (i) $\phi \rightarrow \eta \mathrm{e}^{+} \mathrm{e}^{-}$, (ii) $\mathrm{e}^{+} \mathrm{e}^{-} \rightarrow \mu^{+} \mu^{-} \gamma$, (iii) $\mathrm{e}^{+} \mathrm{e}^{-} \rightarrow \mathrm{e}^{+} \mathrm{e}^{-} \gamma$, and (iv) $\mathrm{e}^{+} \mathrm{e}^{-} \rightarrow \mu^{+} \mu^{-} \not$.

$$
\text { 2.1. The } \mathrm{e}^{+} \mathrm{e}^{-} \rightarrow \eta \mathrm{e}^{+} \mathrm{e}^{-} \text {final state }
$$

As for the process $\mathrm{e}^{+} \mathrm{e}^{-} \rightarrow \eta \mathrm{U}$, with dark photon decays in $\mathrm{e}^{+} \mathrm{e}^{-}$, the papers of Refs. $[8,9]$ have been published. From a sample of $\approx 6 \times 10^{9} \phi$ mesons $\left(1.7 \mathrm{fb}^{-1}\right)$ we select $\phi \rightarrow \eta \mathrm{e}^{+} \mathrm{e}^{-}$Dalitz decays, with $\eta \rightarrow \pi^{+} \pi^{-} \pi^{0}$ and $\eta \rightarrow \pi^{0} \pi^{0} \pi^{0}$. Event selection requires two opposite-charge particles from the interaction region and six prompt clusters in the calorimeter, or two clusters and two opposite-charge particles, with invariant mass compatible with the $\eta$ mass. The contamination from $\phi \rightarrow \eta \gamma$ with photon conversion on the beam pipe or drift chamber wall (DCW) is suppressed by cuts on $m_{\mathrm{ee}}$ and on the position of the closest approach point (PCA) of the track to both, the beam pipe, and the DCW. We obtain $\approx 3.0 \times 10^{4}\left(1.3 \times 10^{4}\right)$ events in the neutral (charged) $\eta$ decay channel with a contamination level kept below 3 (2)\%. Residual background is evaluated from a fit to data using MC-predicted shapes. After background subtraction, consistent $m_{\mathrm{ee}}$ distributions from events with neutral and charged $\eta$ decay are obtained. The dark photon search is performed in $5 \mathrm{MeV}$ bins, from $5-450$ $\mathrm{MeV}$, separately for neutral and charged $\eta$ decays. Combined $\mathrm{CL}_{\mathrm{S}}$ upper limits from the two samples are shown in Fig. 1, left. We obtained an excluded region at 90\% CL in the $m_{\mathrm{U}^{-}} \epsilon^{2}$ plane that rules out values of the kinetic mixing parameter above $8 \times 10^{-6}$ in the mass range 50-210 MeV. 

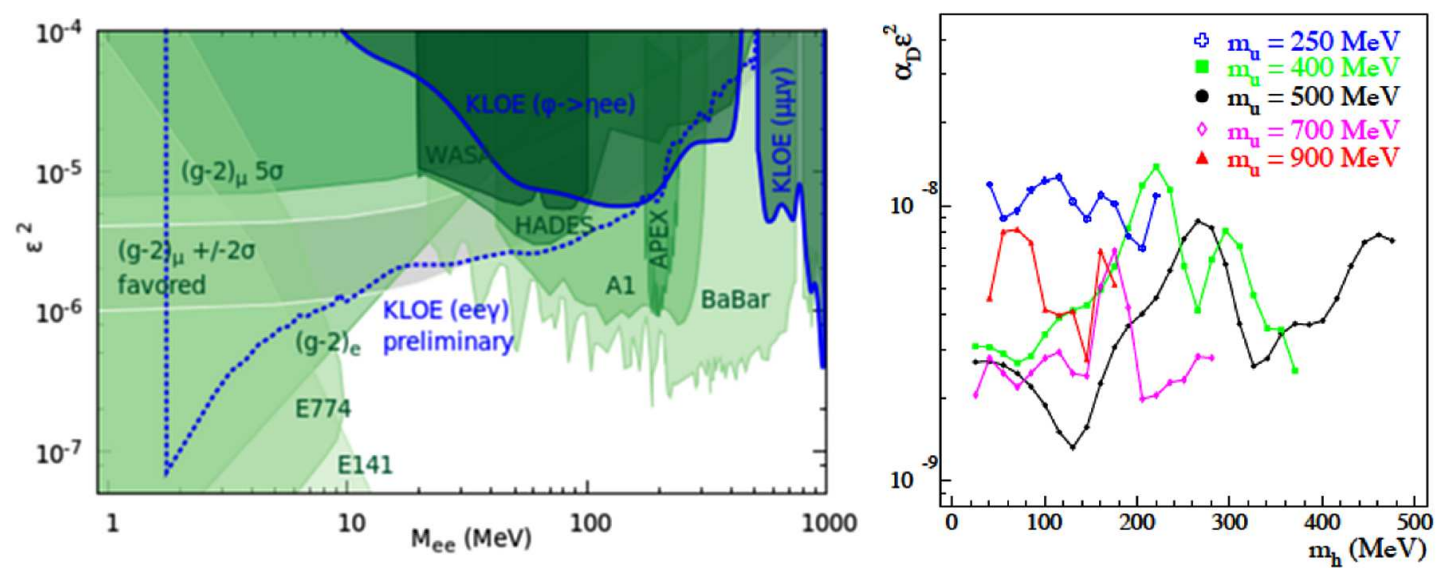

Fig. 1. Left: Exclusion plot at $90 \% \mathrm{CL}$ in the $m_{\mathrm{U}}-\epsilon^{2}$ plane obtained from the analysis of the $\mathrm{e}^{+} \mathrm{e}^{-} \rightarrow \mathrm{e}^{+} \mathrm{e}^{-} \eta$, $\mathrm{e}^{+} \mathrm{e}^{-} \rightarrow \mu^{+} \mu^{-} \gamma$, and $\mathrm{e}^{+} \mathrm{e}^{-} \rightarrow \mathrm{e}^{+} \mathrm{e}^{-} \gamma$ final states. Right: Preliminary upper limits on $\alpha_{\mathrm{D}} \times \epsilon^{2}$ at $90 \%$ CL, as a function of $m_{\mathrm{h}^{\prime}}$ and for different values of $m_{\mathrm{U}}$, from the analysis of the $\mu^{+} \mu^{-} E^{2}$ final state.

\subsection{The $\mathrm{e}^{+} \mathrm{e}^{-} \rightarrow \mu^{+} \mu^{-} \gamma$ final state}

The reaction $\mathrm{e}^{+} \mathrm{e}^{-} \rightarrow \gamma \mathrm{U}$, with dark photon decays to muon pairs, was studied on the sample used for the measurement of the ratio $R=\sigma\left(\mathrm{e}^{+} \mathrm{e}^{-} \rightarrow\right.$ $\left.\pi^{+} \pi^{-}\right) / \sigma\left(\mathrm{e}^{+} \mathrm{e}^{-} \rightarrow \mu^{+} \mu^{-}\right)$, exploiting the precision MC simulation and the data analysis chain of the QED process $\mathrm{e}^{+} \mathrm{e}^{-} \rightarrow \mu \mu \gamma$ published in Ref. [10]. A $\mu \mu \gamma$ candidate must have two tracks of opposite charge from the interaction region. We require two tracks at large polar angle, $50^{\circ}<\theta<130^{\circ}$, and one undetected photon. The separation between tracks and photon regions greatly reduces the contamination from both, the resonant $\mathrm{e}^{+} \mathrm{e}^{-} \rightarrow \phi \rightarrow \pi^{+} \pi^{-} \pi^{0}$, and FSR processes, $\mathrm{e}^{+} \mathrm{e}^{-} \rightarrow \pi^{+} \pi^{-} \gamma_{\mathrm{FSR}}$ and $\mathrm{e}^{+} \mathrm{e}^{-} \rightarrow \mu^{+} \mu^{-} \gamma_{\mathrm{FSR}}$. Electron, pion and muon pairs are identified by the mass obtained assuming an $\mathrm{e}^{+} \mathrm{e}^{-} \rightarrow x^{+} x^{-} \gamma$ process. In addition, a pseudo-likelihood estimator based on time-of-flight and other calorimeter information is used for improving on the separation of electrons from pions and muons. Residual contamination and analysis efficiency are evaluated from MC simulations that have been controlled with real data samples. The differential $\mu \mu \gamma$ cross-section is in excellent agreement with expectations from the NLO PHOKHARA generator [11]. The exclusion plot for $\mathrm{e}^{+} \mathrm{e}^{-} \rightarrow \mathrm{U} \gamma \rightarrow \mu^{+} \mu^{-} \gamma$ is obtained using the $\mathrm{CL}_{\mathrm{S}}$ technique. It covers the mass region $520<m_{\mathrm{U}}<980 \mathrm{MeV}$ (Fig. 1, left), with upper limits at $90 \%$ CL on $\epsilon^{2}$ from $8.6 \times 10^{-7}$ to $1.6 \times 10^{-5}$.

\subsection{The $\mathrm{e}^{+} \mathrm{e}^{-} \rightarrow \mathrm{e}^{+} \mathrm{e}^{-} \gamma$ final state}

We are studying the $\mathrm{e}^{+} \mathrm{e}^{-} \rightarrow \mathrm{e}^{+} \mathrm{e}^{-} \gamma$ final state, searching for an excess in the $\mathrm{e}^{+} \mathrm{e}^{-}$invariant mass spectrum that could signal dark photon production in $\mathrm{e}^{+} \mathrm{e}^{-} \rightarrow \mathrm{U} \gamma$ processes. We select events with finalstate electron, positron, and photon, all emitted at large angle $(|\cos \theta|<0.57)$, such that they have an associated cluster in the calorimeter barrel. As in the case of $\mu \mu \gamma$ analysis, the electrons are separated from muons and pions using the $m_{\text {track }}$ variable obtained assuming an $\mathrm{e}^{+} \mathrm{e}^{-} \rightarrow x^{+} x^{-} \gamma$ process. The sum of all background sources, $\mathrm{e}^{+} \mathrm{e}^{-} \rightarrow \mu^{+} \mu^{-} \gamma, \mathrm{e}^{+} \mathrm{e}^{-} \rightarrow \pi^{+} \pi^{-} \gamma, \mathrm{e}^{+} \mathrm{e}^{-} \rightarrow \gamma \gamma$, and $\mathrm{e}^{+} \mathrm{e}^{-} \rightarrow \phi \rightarrow \pi^{+} \pi^{-} \pi^{0}$ is typically less than $1 \%$, and none of the contributions is peaked in the electron pair invariant mass distribution. After background subtraction, the $m_{\mathrm{ee}}$ spectrum is in good agreement with full Monte Carlo simulation based on the Babayaga [12] generator. The limit on the number of dark photons as a function of the $m_{\mathrm{ee}}$ invariant mass is calculated using the $\mathrm{CL}_{\mathrm{S}}$ technique and evaluating the background in each $m_{\text {ee }}$ bin by a fit to the sidebands. The preliminary evaluation of the exclusion region at $90 \%$ CL for the kinetic mixing parameter $\epsilon^{2}$ as a function of the dark photon mass is shown in Fig. 1, left. Upper limits on $\epsilon^{2}$ range from $10^{-7}-10^{-5}$, increasing with the dark photon mass in the interval between 2 and $250 \mathrm{MeV}$.

\subsection{The $\mathrm{e}^{+} \mathrm{e}^{-} \rightarrow \mu^{+} \mu^{-} E$ final state}

The search for the process $\mathrm{e}^{+} \mathrm{e}^{-} \rightarrow \mathrm{Uh}^{\prime}$ with $\mathrm{U} \rightarrow$ $\mu^{+} \mu^{-}$and h' invisible is based on $1.65 \mathrm{fb}^{-1}$ of integrated luminosity at the $\phi$ peak and $0.2 \mathrm{fb}^{-1}$ at $1 \mathrm{GeV}$. Events with (i) only two opposite charge tracks from the interaction region, with (ii) associated clusters in the barrel calorimeter, (iii) total momentum greater than $450 \mathrm{MeV}$, and (iv) missing momentum greater than $40 \mathrm{MeV}$ are selected requiring in addition that (v) the particle momentum is below $460 \mathrm{MeV}$, and (vi) the opening angle between tracks has $\left|\cos \theta_{12}\right|<0.8$. These criteria, together with rejection of events with prompt clusters without any associated track (calorimeter veto), cut mostly of the QED background. Muon discrimination against electrons is based on time-of-flight and other calorimeter information, selecting $85 \%$ of muon pairs and only $0.1 \%$ of electron pairs. Remaining background in the on-peak sample, mostly from $\phi$ decays to charged kaons with early kaon decay to $\mu \nu$, is suppressed by a factor 
of 80 with cuts on vertex quality and position. Final samples of 15278 events in the on-peak and 783 events in the off-peak data have been divided into 53 bins of the $m_{\mu \mu}-m_{\text {miss }}$ plane, and separately studied. We have chosen a bin width of $5 \mathrm{MeV}$ in the $m_{\mu \mu}$ range from $2 \times m_{\mu}-1000 \mathrm{MeV}$, and a variable binning of 15,30 and $50 \mathrm{MeV}$ in the $m_{\text {miss }}$ range from $20-500 \mathrm{MeV}$, to keep more than $90 \%$ of the signal in one bin only. As no evidence of the dark higgstrahlung process was found, 90\% CL Bayesian upper limits are derived bin by bin, for the on-peak and off-peak samples, separately. Combined results are almost everywhere dominated by the on-peak sample, with the exception of those regions with the highest background level from $\phi$ decays. Preliminary upper limits on $\alpha_{\mathrm{D}} \times \epsilon^{2}$ range from $10^{-9}$ to $10^{-8}$ as a function of $m_{\mathrm{h}^{\prime}}$ and $m_{\mathrm{U}}$ (Fig. 1, right).

\section{References}

[1] B. Batell, M. Pospelov, A. Ritz, Phys. Rev. D 80, 095024 (2009).

[2] N. Arkani-Hamed, N. Weiner, J. High Energy Phys. 12, 104 (2008).

[3] J. Beatty, A. Bhattacharyya, C. Bower, S. Coutu, M.A. DuVernois, S. McKee, S.A. Minnick, D. Müller, J. Musser, S. Nutter, A.W. Labrador, M. Schubnell, S. Swordy, G. Tarlé, A. Tomasch, Phys. Rev. Lett. 93, 241102 (2004).
[4] O. Adriani, et al. (PAMELA), Nature 458, 607 (2009).

[5] L. Accardo, et al. (AMS), Phys. Rev. Lett. 113, 121101 (2014).

[6] O. Adriani, et al. (PAMELA), Phys. Rev. Lett. 102, 051101 (2009).

[7] B. Batell, M. Pospelov, A. Ritz, Phys. Rev. D 79, 115008 (2009).

[8] F. Archilli, et al. (KLOE-2), Phys. Lett. B 706, 251 (2012).

[9] D. Babusci, et al. (KLOE-2), Phys. Lett. B 720, 111 (2013).

[10] D. Babusci, et al. (KLOE), Phys. Lett. B 720, 336 (2013).

[11] H. Czyz, A. Grzelinska, J. H. Kuhn, G. Rodrigo, Eur. Phys. J. C 39, 411 (2005).

[12] L. Barze, G. Balossini, C. Bignamini, C. M. Carloni Calame, G. Montagna, O. Nicrosini, F. Piccinini, Eur. Phys. J. C 71, 1680 (2011). 